\title{
A Human Epithelium-Specific Vector Optimized in Rat Pneumocytes for Lung Gene Therapy
}

\author{
DAVID R. KOEHLER, YU-HUA CHOW, JONATHAN PLUMB, YANXIA WEN, BIJAN RAFII, \\ ROSETTA BELCASTRO, MARTIN HAARDT, GERGELY L. LUKACS, MARTIN POST, \\ A. KEITH TANSWELL, AND JIM HU
}

Programmes in Lung Biology Research [D.R.K., Y.H.C., J.P., Y.W., B.R., R.B., M.P., A.K.T., J.H.] and

Cell Biology [M.H., G.L.L.], and the Medical Research Council Group in Lung Development [D.R.K., M.P., A.K.T.], The Hospital for Sick Children, 555 University Ave., Toronto, Ontario M5G 1X8, Canada

\begin{abstract}
ABSTR
Gene therapy vectors based on mammalian promoters offer
the potential for increased cell specificity and may be less
susceptible than viral promoters to transcription attenuation by
host cytokines. The human cytokeratin 18 (K18) gene is naturally
expressed in the lung epithelia, a target site for gene therapies to
treat certain genetic pediatric lung diseases. Our original vector
based on the promoter and 5' control elements of K18 offered
excellent epithelial cell specificity but relatively low expression
levels compared with viral promoters. In the present study, we
found that adding a stronger SV40 poly(A) signal boosted pri-
mary rat lung epithelial cell expression but greatly reduced cell
specificity. Addition of a $3^{\prime}$ portion of the K18 gene to our vector
as a $3^{\prime}$ untranslated region (UTR) improved epithelial cell-
specific expression by reducing expression in lung fibroblasts.
The effect of the $3^{\prime}$ UTR was not related to gross differences in
cell-specific splicing. A deletion variant of this UTR further
\end{abstract}
increased lung epithelial cell expression while retaining some cell specificity. These data illustrate the possibilities for using $3^{\prime}$ UTR to regulate cell-specific transgene expression. Our improved K18 vector should prove useful for pediatric lung gene therapy applications. (Pediatr Res 48: 184-190, 2000)

K18, cytokeratin 18
CF, cystic fibrosis
CFTR, cystic fibrosis transmembrane conductance regulator
SEAP, secreted alkaline phosphatase
CMV, cytomegalovirus
UTR, untranslated region
IFN- $\boldsymbol{\gamma}$, interferon- $\gamma$
TNF- $\boldsymbol{\alpha}$, tumor necrosis factor- $\alpha$

The epithelial surface of the airways presents an attractive opportunity for the topical delivery of gene-based treatments for pediatric lung diseases. CF and $\alpha_{1}$-antitrypsin deficiency, the two most common fatal monogenic lung disorders in the Caucasian population, have garnered much attention as possible targets for lung gene therapy $(1,2)$. Other lung disorders that may someday benefit from gene therapies include genetic surfactant protein B deficiency (3) and inflammatory lung diseases (2). CF is caused by a defect in the gene encoding CFTR, a chloride channel residing in the epithelium of the lungs and other organs $(4,5)$. CF affects multiple organ systems, but it is the lung disease, characterized by thick mucous, persistent bacterial infection, and inflammation, that causes the majority of morbidity and mortality associated with

Received November 3, 1999; accepted March 13, 2000

Correspondence: Jim Hu, M.D., Lung Biology Research, Hospital for Sick Children, 555 University Ave., Toronto, Ontario M5G 1X8, Canada.

Supported by a Research and Development Program Grant from the Canadian Cystic Fibrosis Foundation (SPARX II), grants from the Medical Research Council of Canada, the Ontario Thoracic Society, the Hospital for Sick Children Research Foundation, and a Fellowship to D.R.K. from the Medical Research Council of Canada.
CF $(5,6)$. In theory, early intervention to deliver a functional CFTR gene to just 5 to $10 \%$ of the defective lung cells of CF patients could lead to an improvement in the lung airway surface fluid and an improved prognosis $(7,8)$. Attempts at human gene therapy proved the feasibility of this approach (9), but challenges remain before $\mathrm{CF}$ gene therapy becomes a reality.

Viral vectors such as those derived from human adenovirus can efficiently deliver DNA to cells, but host immune responses to both the virus and transgene are problematic (10, 11). Complexes of DNA and cationic liposome have been widely tested and have the advantages of relatively low toxicity, low immunogenicity, and ease of preparation (12) but are currently inefficient as DNA delivery vehicles $(13,14)$. It may be possible to counter this problem by choosing a strong expression vector to drive the production of CFTR. It may also be desirable to have expression that is long-lasting, which could lower the frequency of applications, reducing both adverse effects and expense. Controlling the cell specificity of CFTR expression could be important for reducing gene therapy side effects. In the lung, CFTR is expressed in the airway 
epithelial and submucosal gland cells (15). Inappropriate CFTR expression in cultured cells can cause growth abnormalities $(16,17)$, but clinical effects, if any, are not known.

To maximize gene expression, many gene therapy vectors use strong viral promoters such as the widely used CMV immediate-early promoter/enhancer (18). Although high expression levels can be achieved in cultured cells by use of viral promoters, they may not be a good choice for sustained gene expression for lung gene therapy. It has been shown that transcription from viral promoters, including CMV, is downregulated by host cytokines such as IFN- $\gamma$ and TNF- $\alpha$ in a phenomenon known as "promoter attenuation" (19). These cytokines are induced by adenoviral and DNA:liposome gene therapy delivery systems $(20,21)$.

We sought to avoid these pitfalls and improve the duration and specificity of transgene expression both in vitro and ultimately in vivo by using a nonviral promoter naturally active in lung epithelial cells. Human K18 is expressed specifically in the epithelial cells of internal organs (22). Its expression is regulated by positive and negative elements upstream of the promoter and in the first intron and exon 6 (23-25). Using a vector constructed with the K18 promoter, first intron, and upstream regulatory elements, we were able to drive epithelialspecific gene expression in transgenic mice and in transiently transfected cultured human cells (26). Although our firstgeneration $\mathrm{K} 18$ vector demonstrated promising long-term transgene expression and excellent epithelial cell specificity in cultured human cells, short-term expression was low in relation to a CMV-based vector. In the present study, we have improved the level of expression from our K18-based vector while retaining its epithelial cell specificity through the use of a $3^{\prime}$ UTR derived from K18.

\section{METHODS}

Plasmids. pK18SEAP-EpA was previously described as K18EpilongSEAP (26) and contains the promoter and $2.5-\mathrm{kb}$ upstream genomic sequence from the human K18 gene plus the first intron of K18, before the start of the SEAP gene (pSEAP, Tropix, Bedford, MA, U.S.A.). pCMVSEAP-EpA was constructed by cloning the CMV immediate-early promoter/ enhancer from pCEP4 (Invitrogen) into pSEAP. After the SEAP gene, both these vectors include the SV40 $t$ intron and SV40 minimal (237 nt) early poly(A) signal. These were replaced with the SV40 late poly(A) signal from pMP6a (27) (originally from pREP4, Invitrogen, Carlsbad, CA, U.S.A.) to create pK18SEAP-LpA and pCMVSEAP-LpA.

A 1-kb region of the K18 gene from nucleotides 5521-6520, referred to here as K18 native 3' (or K18n3'), was PCR cloned from human genomic DNA. This region comprises K18 exon 6 , intron 6 , exon 7 , and 272 nt of untranslated downstream sequence including the K18 poly(A) signal. When added to our SEAP vectors, replacing the SV40 poly(A) signal, this DNA segment functioned as a $3^{\prime}$ UTR with a K18 poly(A) signal. Deletions of this region were constructed as depicted in Figure $3 A$, using the $K p n \mathrm{I}$ and $P m l \mathrm{I}$ restriction sites indicated.

pK18mTElacZ-K18i6x7pA was created by removing the SV40 medium-length (512 nt) early poly(A) signal of
pK18mTElacZ (26) and replacing it with the K18i6x7pA UTR. The parental K18 vector contained a mutation $(\mathrm{m})$ of the K18 first intron $3^{\prime}$ splice acceptor site to reduce exon skipping events and a translational enhancer (TE) sequence from alfalfa mosaic virus (28) placed immediately upstream of the lacZ start codon (26). The bacterial $\beta$-galactosidase gene included a $5^{\prime}$ nuclear localization signal (NLS) from the SV40 large T protein. pMP6aTElacZ was created by inserting the TE and $\beta$-galactosidase gene of $\mathrm{pK} 18 \mathrm{mTElacZ}$ into the multicloning site of pMP6a (27).

pK18mTECFTR-K18i6x7pA was created by removing the SV40 minimal early poly(A) signal of pK18mTECFTR (26) and replacing it with the $-\mathrm{K} 18 \mathrm{i} 6 \mathrm{x} 7 \mathrm{pA}$ UTR. The parental K18 vector contains the $\mathrm{K} 18$ first intron mutation and TE mentioned above. Additionally, a silent mutation was placed in the CFTR cDNA to eliminate cryptic splicing events documented between K18 intron 1 and CFTR (26). Epitope-tagged versions of
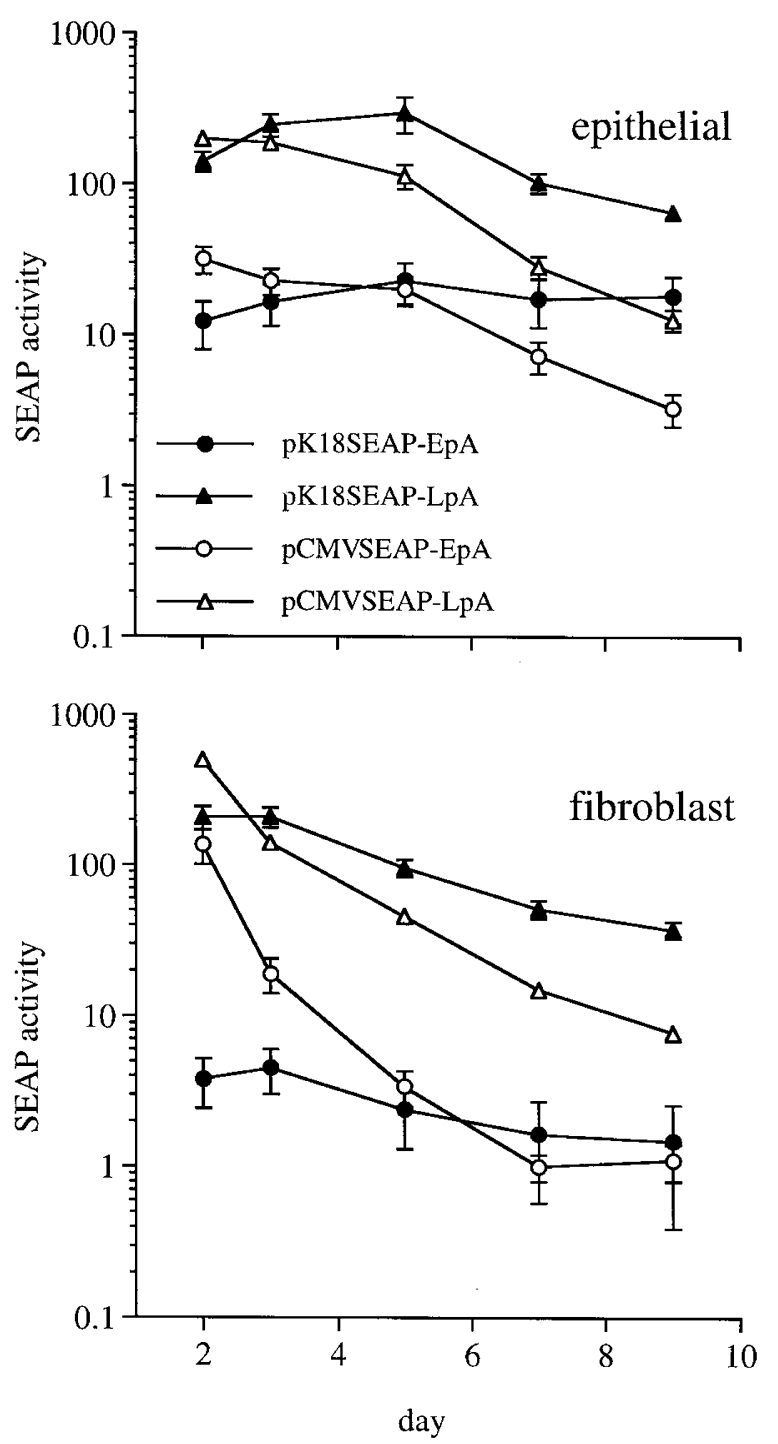

Figure 1. Effect of changing polyadenylation signals on expression. SEAP activity was measured in culture medium from primary rat pneumocytes transfected with pK18SEAP or pCMVSEAP with either the SV40 minimal early poly(A) signal $(E p A)$ or SV40 late poly(A) signal $(L p A)$. Data are presented in thousands of relative light units as mean \pm SEM; $n=4-16$. 
these vectors were created by using a CFTR cDNA that had the VSV-G coding sequence added to place the tag at the protein's C-terminus (pCFTag, Z. Lu). To correct the splicing defect arising from a cryptic $5^{\prime}$ donor site in the VSV-G coding sequence, this sequence was changed from TACACCGACATCGAGATGAACCGGCTGGGCAAG to TACACCGACATCGAGATGAACCGGCTtGGgAAa by mutagenic primer PCR.

Tissue culture and reporter gene assays. COS- 1 and A549 cells were maintained as recommended by the American Type Culture Collection. Primary cultures of rat lung epithelial and fibroblast cells were isolated from gestational d 19-20 rat fetuses and maintained in culture as described (29). Cell populations isolated using this method are approximately $90 \%$ pure $(29,30)$. One day after isolation, $0.5 \times 10^{6}$ cells were seeded per $35-\mathrm{mm}$ well in a 6 -well plate and were transfected $18 \mathrm{~h}$ later (at 50 to $80 \%$ confluence) with $1 \mu \mathrm{g}$ of plasmid DNA premixed with $12 \mu \mathrm{g}$ of lipofectAMINE (as recommended by the manufacturer, GIBCO/BRL, Rockville, MD, U.S.A.) in duplicate culture wells. Cell cultures became confluent and contact growth-inhibited by the first assay point $(2 \mathrm{~d}$ posttransfection). The $2-\mathrm{mL}$ culture medium was changed $24 \mathrm{~h}$ before each assay point. The 24-h accumulation of SEAP activity was assayed in $30 \mu \mathrm{L}$ of media by using the Phospha-Light chemi-
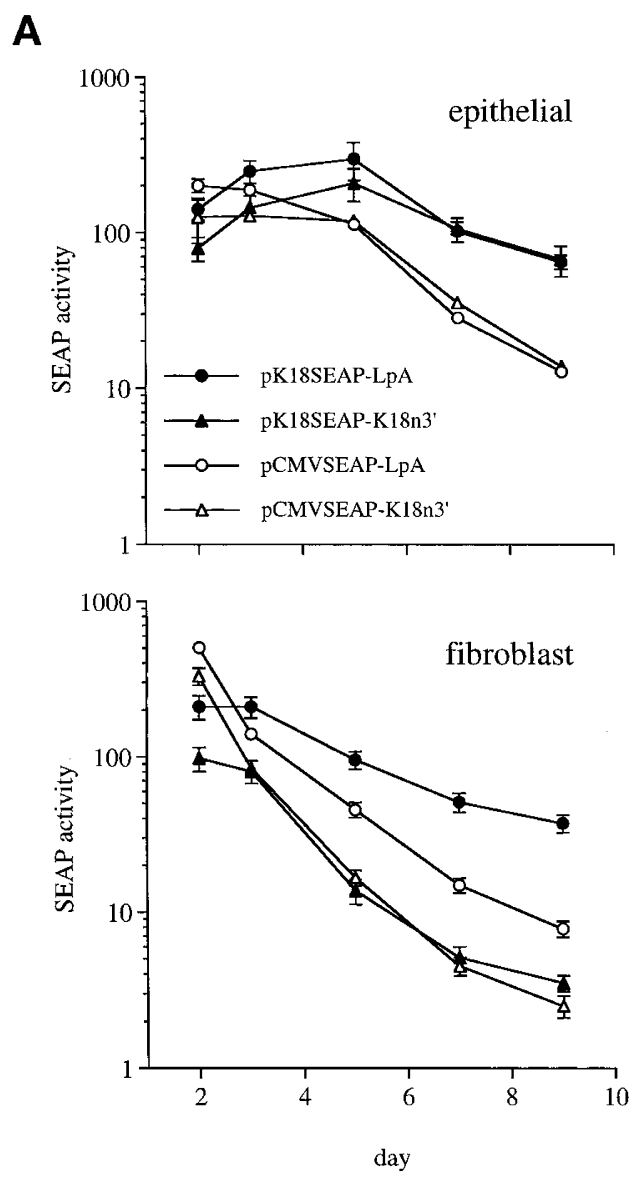

luminescent system (Tropix, Bedford, MA, U.S.A.) and measured on a microplate luminometer (E.G.\&G. Berthold LB96V, Bad Wildbad, Germany). Data are expressed as thousands of relative light units after subtracting a negative control. To correct for variation in the transfection capacity of different batches of primary cells, pCMVSEAP was used as a transfection control in separate duplicate culture wells (we found that using internal control plasmids caused distortion of the data). Each data set from different cell batches was then normalized to the average value for pCMVSEAP expression at $2 \mathrm{~d}$ posttransfection (the first time point assayed) for that cell batch.

$\beta$-galactosidase activity in cell lysates was assayed using the Galacto-Light chemiluminescent system (Tropix) and measured on a microplate luminometer (E.G.\&G. Berthold LB96V). Values are expressed as thousands of relative light units after normalizing for lysate protein content and subtracting a negative control.

Reverse transcriptase-PCR (RT-PCR) and Western blot. Total RNA was isolated from cells $3 \mathrm{~d}$ after transfection, treated with DNaseI to remove residual plasmid DNA, repurified, and reverse transcribed with the primer ATTGGCCTCCTGCTCCCAAAGG in the K18n3' region [after exon 7 and before the poly(A) cleavage site]. PCR to detect splicing of K18 intron 6 in pK18SEAP-K18n3' was performed with the

\section{B}
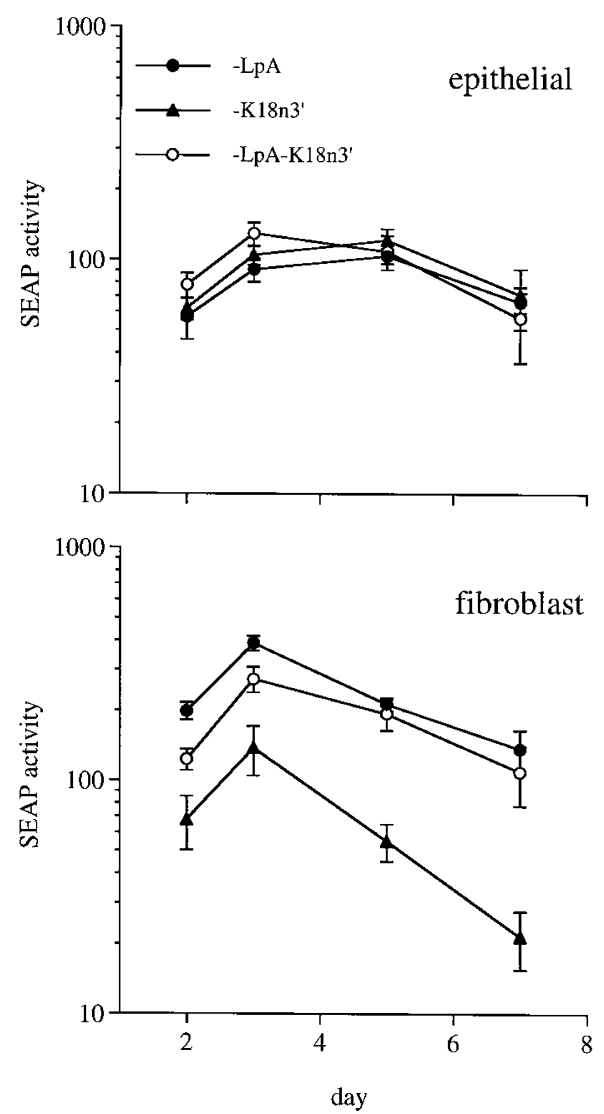

Figure 2. (A) Addition of a $3^{\prime}$ region of the K18 gene as a $3^{\prime}$ UTR. SEAP activity was measured in culture medium from rat pneumocytes transfected with pK18SEAP or pCMVSEAP with either the SV40 late poly (A) signal $(L p A)$ or a $3^{\prime}$ region of the human K18 gene $\left(K 18 n 3^{\prime}\right) ; n=6-16$. (B) Addition of an SV40 late poly(A) signal $(L p A)$ upstream of the K18n3' UTR. SEAP activity was measured in culture medium from rat pneumocytes transfected with K18 vectors with either the SV40 late poly(A) signal $(L p A), \mathrm{K} 18 \mathrm{n} 3^{\prime}$ UTR, or an SV40 late poly(A) signal $(L p A)$ preceding the K18n3' region; $n=4-7$. 
primer GAAACGGTCCAGGCTATGTGCTC in the SEAP coding region and GGTGTCATTGGTCTCAGACACCAC in the $\mathrm{K} 18 \mathrm{n} 3$ ' region.

Whole lysates of COS-1 cells were prepared in RIPA buffer as described (31) $48 \mathrm{~h}$ after transfection with CFTR vectors. After SDS/PAGE of cell extracts containing $100 \mu \mathrm{g}$ of total protein per lane and Western transfer, the blot was probed with the C-terminus-specific CFTR MAb L12B4 (32).

\section{RESULTS}

Polyadenylation signals and cell specificity. As suggested from earlier work with cell lines (26), our K18-based vectors demonstrated excellent long-term transgene expression in primary rat pneumocytes (Fig. 1). Replacing the SV40 minimal early poly(A) signal with the SV40 late poly(A) signal in both K18- and CMV-based vectors increased SEAP expression greatly, as expected (33). In epithelial cells, changing the poly(A) signal increased gene expression up to 8-fold for the CMV-based vector and approximately 40-fold for the K18based vector. In fibroblasts, changing the poly(A) signal increased expression up to 15-fold for the CMV-based vector but up to 100-fold for the K18-based vector. This resulted in a large relative decrease in epithelial cell specificity of the K18-based vector. In preliminary experiments, we found that using either the SV40 full-length early (988 nt) or bovine $\mathrm{GH}$ poly(A) signals with the K18-based vector resulted in levels of transgene expression similar to that of the SV40 late poly(A) signal, with a similar loss of epithelial cell specificity.

A 3' region of human $K 18$ enhances epithelial cell specificity. We tested a $3^{\prime}$ genomic region of the human $\mathrm{K} 18$ gene comprising exon 6 , intron 6 , exon 7 , the poly(A) signal, and $3{ }^{\prime}$ flanking sequence for its effect on expression and cell specificity. This region was selected because it contains control elements found in exon 6 of the K18 gene (25). We added it to our K18 vector as a 3' UTR, replacing the viral poly(A) signal, to mimic the $3^{\prime}$ structure of the natural human K18 transcription unit. When this region (n3') was added, epithelial cell expression was comparable in level and duration to the K18 vector with the SV40 late poly(A) signal whereas fibroblast expression was reduced, and this difference increased at later times after transfection (Fig. 2, $A$ and $B$ ). The K18n3' UTR also improved the epithelial cell specificity of a CMV-based vector (Fig. $2 A$ ).

To determine whether the reduction in fibroblast expression was due to events occurring before or after transcription, we placed an SV40 late poly(A) signal upstream of the K18 n3' region (but downstream of SEAP) in our K18 vector. This is expected to eliminate any effects of RNA sequences downstream of the viral poly $(\mathrm{A})$ signal after polyadenylation processing. When the viral poly(A) signal preceded the K18 n3' region, expression was similar to that of the SV40 late poly(A) signal alone in both epithelial and fibroblast cells (Fig. 2B). These data suggest that the effect of the unencumbered K18 n3' region on reducing expression in fibroblasts is due to events occurring after transcription.

Deletions of the K18 n3' UTR. We performed deletions of the K18 n3' UTR (Fig. 3A) to identify the region(s) responsible
A
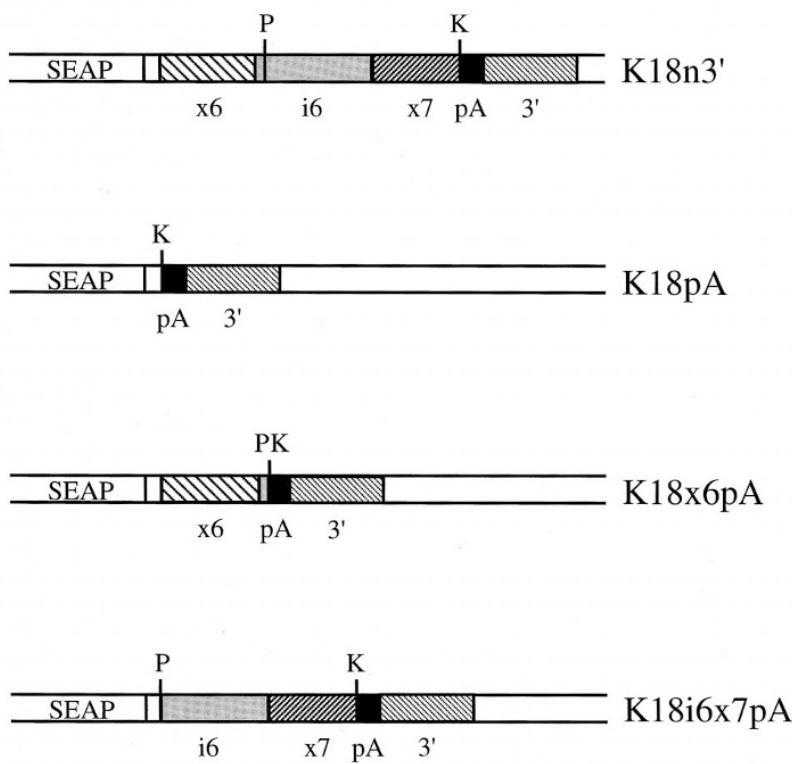

B
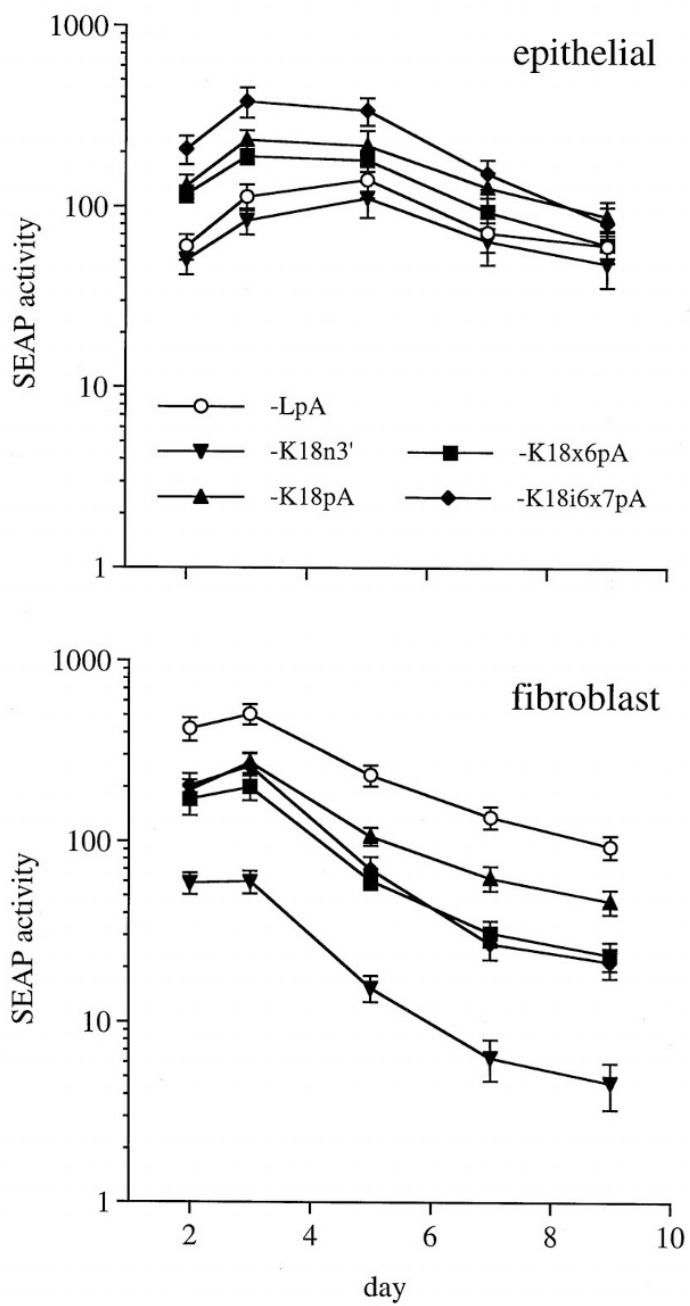

Figure 3. $(A)$ Deletions of the K18n $3^{\prime}$ UTR. The region consists of K18 exon $6(x 6)$, intron $6(i 6)$, exon $7(x 7)$, the poly(A) signal $(p A)$, and downstream flanking sequence $\left(3^{\prime}\right)$. KpnI $(K)$ and $P m l \mathrm{I}(P)$ restriction sites are indicated. $(B)$ Effect of K18n3' UTR deletions on expression. SEAP activity measured in culture medium from rat pneumocytes transfected with $\mathrm{K} 18$ vectors with either the SV40 late poly(A) signal ( $L p A)$, the wild-type K18n3' UTR, or deletions of the K18n3' UTR; $n=6-10$. 
for reducing transgene expression in fibroblasts. K18 exon 6 was of particular interest because it contains a DNaseI hypersensitive region and AP-1 binding site. Deleting intron 6 and exon 7 to leave exon 6 and the K18 poly(A) signal (-K18x6pA) resulted in a similar level of epithelial expression compared with the K18 poly(A) signal alone and a reduction in fibroblast expression at later times (Fig. $3 B$ ). Deleting exon 6 to leave intron 6, exon 7, and the K18 poly(A) signal (-K18i6x7pA) resulted in a 4-fold increase in epithelial expression in the first $3 \mathrm{~d}$ compared with -K18n3' and a pattern of fibroblast expression similar to that of $-\mathrm{K} 18 \mathrm{x} 6 \mathrm{pA}$. When we replaced the SV40 late poly(A) signal of a CMV-based vector with the $-\mathrm{K} 18 \mathrm{i} 6 \mathrm{x} 7 \mathrm{pA}$ UTR, expression in lung epithelial cells doubled whereas fibroblast expression was halved (data not shown).

Effect of intron 6 splicing on transgene expression. Although the $-\mathrm{K} 18 \times 6 \mathrm{pA}$ and $-\mathrm{K} 18 \mathrm{i} 6 \times 7 \mathrm{pA}$ constructs together contain all the DNA sequences present in K18n3', the total decrease in fibroblast expression (relative to pK18SEAP-LpA) seen with both vectors is less than half that seen with the intact K18n3' (Fig. 3B). Because only the intact K18n3' has a spliceable intron 6 , we sought to examine the role of cellspecific RNA splicing differences on the effect of the K18n3' on cell-specific gene expression. RT-PCR of rat pneumocytes transfected with the K18 vector containing the K18n3' UTR revealed that the correctly spliced product was produced in both epithelial and fibroblast cells (Fig. 4).

Addition of K18i6x7pA 3' UTR to other expression vectors. Based on the knowledge gained from our SEAP reporter gene work, we tested the effect of the K18i6x7pA UTR on the expression characteristics of our K18-based lacZ and CFTR expression vectors. Because both lacZ (34) and CFTR contain potential cryptic $5^{\prime}$ donor sequences in the $3^{\prime}$ regions of their cDNA and the K18i6x7pA UTR contains the $3^{\prime}$ splice acceptor site of intron 6 , there existed a possibility for disruptive cryptic splicing. We, therefore, used RT-PCR to examine the RNA from COS, A549, and primary rat pneumocytes transfected with our K18 lacZ and CFTR vectors. These vectors had no aberrant splicing events in any of the tested cell types (not shown). However, addition of a VSV-G epitope-tagged CFTR sequence to our K18i6x7pA UTR vector produced a destructive splicing event between the sequences coding for the VSV-G tag and the $3^{\prime}$ splice acceptor site of K18 intron 6. Silent mutagenesis of the VSV-G coding sequence to eliminate the cryptic $5^{\prime}$ splice donor sequence successfully eliminated the splicing problem (data not shown).

The vector pK18mTElacZ-K18i6x7pA had improved $\beta$-galactosidase expression compared with its parental vector pK18mTElacZ (Fig. 5A). Expression was enhanced up to 6-fold in epithelial cells and up to 2-fold in fibroblasts, resulting in increased epithelial cell specificity. The increase in fibroblast expression relative to $\mathrm{pK} 18 \mathrm{mTElacZ}$ is an apparent discrepancy with the SEAP data regarding the K18i6x7pA UTR (Fig. 3B). This is due to the fact that the parental vector pK18mTElacZ does not contain the SV40 late poly(A) signal but the weaker SV40 medium-length early poly(A) signal. Compared with the strong expression vector pMP6aTElacZ [based on pMP6a (27)], which contains a CMV promoter/ enhancer, hybrid intron, and SV40 late poly(A) signal, our new pK18mTElacZ-K18i6x7pA vector demonstrated excellent expression in lung epithelial cells with substantially reduced fibroblast expression. In contrast with our work with the SEAP reporter gene, we consistently observed higher levels of lacZ expression in fibroblasts versus epithelial cells with all expression vectors. We and others (35) have noticed that, in general, cationic liposomes tend to transfect fibroblasts more readily than epithelial cells. However, the same liposome (lipofectAMINE) was used in both reporter gene studies. The difference could be related to cell-specific differences in the processing of the two protein products (SEAP is continually excreted whereas the $\beta$-galactosidase we used is NLS-targeted to the nucleus). Alternatively, because the lacZ vectors tested are approximately $20 \%$ larger than the SEAP vectors, lung fibroblasts may be more transfectable with the larger DNA constructs than epithelial cells.

We also tested the ability of the K18i6x7pA UTR to improve our original K18-based CFTR vector (26). Although Western blot is a semiquantitative assay, it appears that a greater amount of mature CFTR protein was produced by

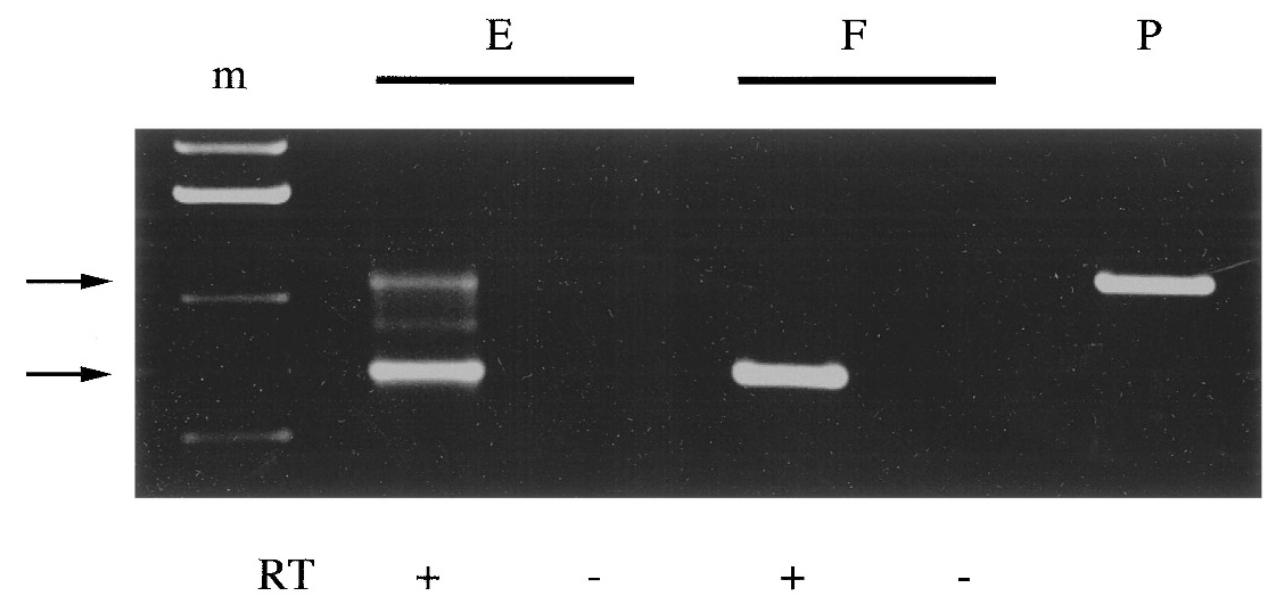

Figure 4. RT-PCR to detect splicing of K18 intron 6 from the K18n $3^{\prime}$ UTR. RT-PCR was performed on RNA from rat lung epithelial $(E)$ and fibroblast $(F)$ cells transfected with pK18SEAP-K18n3'. Reactions were performed with (+) or without $(-)$ RT enzyme $(R T)$. The top and bottom arrows indicate the expected positions of unspliced and spliced products, respectively. PCR of pK18SEAP-K18n3' plasmid DNA $(P)$ indicates the size of the unspliced product. 
pK18mTECFTR-K18i6x7pA than was produced by the original K18 CFTR vector (Fig. $5 B$ ).

\section{DISCUSSION}

We have developed expression vectors using control elements from the human $\mathrm{K} 18$ gene, which is naturally active in lung epithelial cells (22). Our original human K18-based vector offered highly specific and long-lasting epithelial expression in transgenic mice, human cell lines (26), and primary rat pneumocytes (this study), yet expression was relatively low compared with viral promoters. Polyadenylation is a crucial step in RNA processing, mRNA stability, and ultimately gene expression. Because the SV40 minimal early poly(A) signal of our original K18 vector is relatively weak, we replaced it with the strong SV40 late signal. Epithelial cell gene expression was substantially enhanced, but expression in fibroblasts was further increased, resulting in a reduction in epithelial cell specificity. This may reflect differences between epithelial cells and fibroblasts in the responses of their cellular polyadenylation machinery to different viral poly(A) signals.

Exon 6 of the human K18 gene contains a DNaseI hypersensitive region and a functional AP-1 binding site and may be involved in regulating native K18 gene expression (25). We, therefore, tested whether a $1-\mathrm{kb} 3^{\prime}$ genomic fragment of this gene (K18n3'), including exon 6 , could improve the expression characteristics of our K18 vector. We added this region as a $3^{\prime}$ UTR to mimic the 3' structure of the natural K18 transcription unit. A similar approach was successfully developed by Low et al. (36), who used a 3' region of the hGH gene as a $3^{\prime}$ UTR to direct gonadotrophic cell-specific expression in transgenic mice. Replacement of the hGH 3' region with an SV40 poly(A) signal resulted in a loss of gonadotroph-specific expression. We found that addition of the K18n3' UTR to our K18 vector reduced lung fibroblast expression, resulting in an improvement in epithelial cell specificity (up to 11-fold at later times after transfection) compared with the SV40 late poly(A) signal. Lung epithelial cell expression was comparable in level and duration to the K18 vector with the SV40 late poly(A) signal. This effect was also seen to a lesser degree in a CMV-based vector, indicating that the effect is partially independent of the $5^{\prime}$ control elements of our K18 vector.

Untranslated 3' regions can influence gene expression through DNA- and RNA-mediated mechanisms and effects on RNA translation. Deletion analysis of the K18n3' UTR indicated that it contains both positive and negative regulatory elements for transgene expression in lung pneumocytes. Neznanov et al. (25) found that the AP-1 site in exon 6 had a positive effect on expression in F9 embryonic carcinoma cells but not in HR9 endoderm cells. We found that the exon 6 region combined with the $\mathrm{K} 18$ poly(A) signal decreased expression in lung fibroblasts but not in epithelial cells compared with the vector with the $\mathrm{K} 18$ poly $(\mathrm{A})$ signal alone. In the absence of exon 6 (K18i6x7pA), lung fibroblast expression was similarly decreased, whereas epithelial expression was enhanced. However, we added the K18 downstream elements to our vectors as a UTR to mimic the structure of the human K18 transcription unit, whereas Neznanov et al. (25) added
A
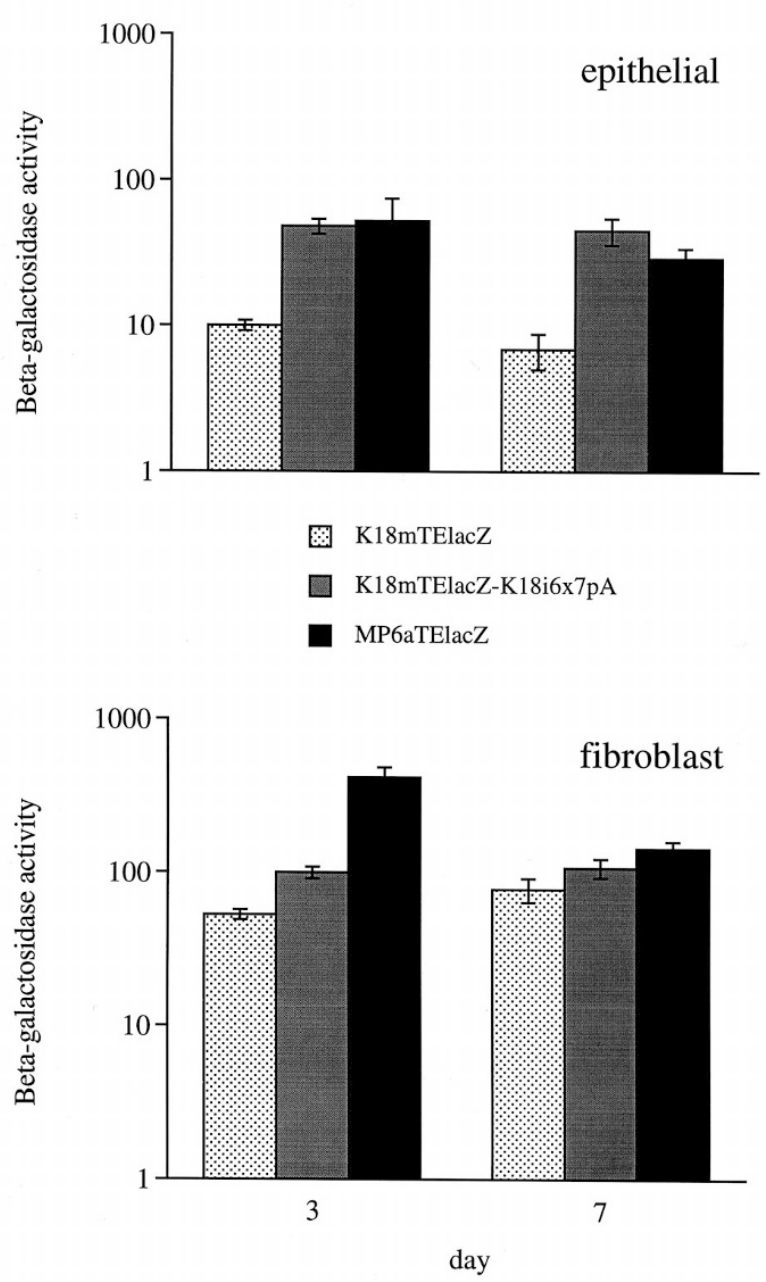

B

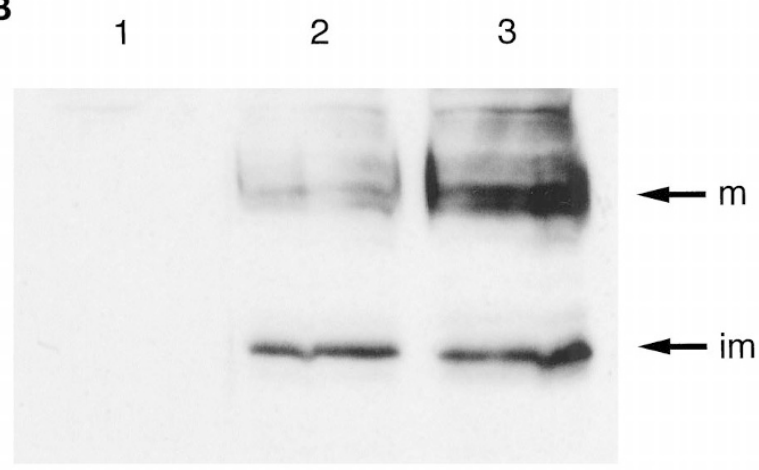

Figure 5. (A) Addition of the K18i6x7pA 3' UTR to our K18-based lacZ vector. $\beta$-Galactosidase activity in cell lysates was measured 3 or $7 \mathrm{~d}$ after transfection of primary rat pneumocytes with K18mTElacZ or K18mTElacZK18i6x7pA; $n=5-10$. Data for a strong CMV-based vector, pMP6aTElacZ, is shown for comparison; $n=2-6$. (B) Western blot to detect CFTR protein expression. COS-1 cells were transfected with 1 ) no DNA, 2) K18mTECFTR, or 3) K18mTECFTR-K18i6x7pA and harvested after $48 \mathrm{~h}$. Arrows indicate the positions of mature $(\mathrm{m})$ and immature $(\mathrm{im})$ CFTR protein.

exon 6 upstream of the K18 promoter to test AP-1 responsiveness. It appears that the K18n3' in our vectors, tested in lung cells, does not act at the DNA level, though. Expression from plasmids with an SV40 poly(A) signal was not affected by the downstream presence of the K18n3'. This also argues against DNA-mediated enhancer/silencer effects or gross differences in 
intracellular plasmid stability due to the K18n3' DNA sequence and suggests that regulation due to the unencumbered K18n3' UTR may occur through an RNA-mediated mechanism.

If mRNA is improperly spliced, the presence of an intron can lead to RNA degradation and lower gene expression (37). We hypothesized that perhaps incorrect splicing of K18 intron 6 in lung fibroblasts was responsible for the reduction in reporter gene activity due to the K18n3' UTR. However, RT-PCR of rat pneumocytes revealed that the K18n3' UTR was correctly spliced in transfected epithelial and fibroblast cells. Even spliced correctly, the K18n3' UTR may act to preferentially reduce the half-life of the mRNA in lung fibroblasts, but this hypothesis remains a topic for future studies.

Using the knowledge gained from our SEAP reporter gene work, we tested the effect of the K18i6x7pA 3' UTR on the expression from our other K18-based vectors. This UTR offers a useful combination of decreased fibroblast expression (up to 4-fold) and enhanced epithelial cell expression (up to 3-fold) compared with the SV40 late poly(A) signal. A lacZ vector incorporating the $\mathrm{K} 18 \mathrm{i} 6 \mathrm{x} 7 \mathrm{pA}$ UTR demonstrated improved epithelial expression and specificity compared with our original (26) K18-based lacZ vector or a strong CMV-based vector. Addition of the K18i6x7pA UTR to our K18-based CFTR vector also proved promising.

In this study, we demonstrated that changing polyadenylation signals of an epithelial-specific human K18-based vector to improve expression levels can have significant effects on cell specificity. We found that a strategy of using additional control elements from K18 as a $3^{\prime}$ UTR was successful in producing high-level epithelial cell expression with good cell specificity. Our new generation K18-based vectors may prove useful for maximizing transgene expression and specificity for gene therapy to treat certain pediatric lung disorders. Although we have focused here on the DNA:liposome delivery route, our expression cassette could be adopted for use with other, possibly more efficient, delivery systems including the latest viral vectors and nonviral strategies that use receptor-mediated endocytosis.

Acknowledgments. The authors thank Dr. Maciej Kuliszewski for sequencing, Dr. Hugh O'Brodovich for critical reading of the manuscript, and the American Type Culture Collection for cell lines. The MAb L12B4 was kindly provided by Dr. Norbert Kartner (Department of Pharmacology, University of Toronto). pCFTag was a gift from Zhan Lu (Department of Genetics, Hospital for Sick Children, Toronto). pMP6a was a gift from Dr. R. Philip (Applied Immune Sciences Inc., Santa Clara, CA, U.S.A.).

\section{REFERENCES}

1. Crystal RG 1992 Gene therapy strategies for pulmonary disease. Am J Med 92:44S$52 \mathrm{~S}$

2. Curiel DT, Pilewski JM, Albelda SM 1996 Gene therapy approaches for inherited and acquired lung diseases. Am J Respir Cell Mol Biol 14:1-18

3. Strayer MS, Guttentag SH, Ballard PL 1998 Targeting type II and Clara cells for adenovirus-mediated gene transfer using the surfactant protein B promoter. Am J Respir Cell Mol Biol 18:1-11

4. Tsui LC 1995 The cystic fibrosis transmembrane conductance regulator gene. Am J Respir Crit Care Med 151:S47-S53

5. Welsh MJ, Tsui LC, Boat TF, Beaudet AL 1995 Cystic fibrosis. In: Scriver CR, Beaudet AL, Sly WS, Valle D (eds) The Metabolic and Molecular Basis of Inherited Disease, Vol III. McGraw-Hill, New York, pp 3799-3809
6. Tizzano EF, Buchwald M 1995 CFTR expression and organ damage in cystic fibrosis. Ann Intern Med 123:305-308

7. Johnson LG, Olsen JC, Sarkadi B, Moore KL, Swanstrom R, Boucher RC 1992 Efficiency of gene transfer for restoration of normal airway epithelial function in cystic fibrosis. Nat Genet 2:21-25

8. Dorin JR, Farley R, Webb S, Smith SN, Farini E, Delaney SJ, Wainwright BJ, Alton EW, Porteous DJ 1996 A demonstration using mouse models that successful gene therapy for cystic fibrosis requires only partial gene correction. Gene Ther 3:797-801

9. Alton EW, Geddes DM, Gill DR, Higgins CF, Hyde SC, Innes JA, Porteous DJ 1998 Towards gene therapy for cystic fibrosis: a clinical progress report. Gene Ther 5:291-292

10. Tripathy SK, Black HB, Goldwasser E, Leiden JM 1996 Immune responses to transgene-encoded proteins limit the stability of gene expression after injection of replication-defective adenovirus vectors. Nat Med 2:545-550

11. Yang Y, Jooss KU, Su Q, Ertl HC, Wilson JM 1996 Immune responses to viral antigens versus transgene product in the elimination of recombinant adenovirusinfected hepatocytes in vivo. Gene Ther 3:137-144

12. Gao X, Huang L 1995 Cationic liposome-mediated gene transfer. Gene Ther 2:710 722

13. Fasbender AJ, Zabner J, Zeiher BG, Welsh MJ 1997 A low rate of cell proliferation and reduced DNA uptake limit cationic lipid-mediated gene transfer to primary cultures of ciliated human airway epithelia. Gene Ther 4:1173-1180

14. Zabner J, Fasbender AJ, Moninger T, Poellinger KA, Welsh MJ 1995 Cellular and molecular barriers to gene transfer by a cationic lipid. J Biol Chem 270:18997-19007

15. Engelhardt JF, Yankaskas JR, Ernst SA, Yang Y, Marino CR, Boucher RC, Cohn JA, Wilson JM 1992 Submucosal glands are the predominant site of CFTR expression in the human bronchus. Nat Genet 2:240-248

16. Stutts MJ, Gabriel SE, Olsen JC, Gatzy JT, O’Connell TL, Price EM, Boucher RC 1993 Functional consequences of heterologous expression of the cystic fibrosis transmembrane conductance regulator in fibroblasts. J Biol Chem 268:20653-20658

17. Schiavi SC, Abdelkader N, Reber S, Pennington S, Narayana R, McPherson JM, Smith AE, Hoppe HT, Cheng SH 1996 Biosynthetic and growth abnormalities are associated with high-level expression of CFTR in heterologous cells. Am J Physiol 270:C341-C351

18. Foecking MK, Hofstetter H 1986 Powerful and versatile enhancer-promoter unit for mammalian expression vectors. Gene 45:101-105

19. Qin L, Ding Y, Pahud DR, Chang E, Imperiale MJ, Bromberg JS 1997 Promoter attenuation in gene therapy: interferon-gamma and tumor necrosis factor-alpha inhibit transgene expression. Hum Gene Ther 8:2019-2029

20. Yang Y, Nunes FA, Berencsi K, Furth EE, Gonczol E, Wilson JM 1994 Cellular immunity to viral antigens limits E1-deleted adenoviruses for gene therapy. Proc Natl Acad Sci USA 91:4407-4411

21. Yew NS, Wang KX, Przybylska M, Bagley RG, Stedman M, Marshall J, Scheule RK, Cheng SH 1999 Contribution of plasmid DNA to inflammation in the lung after administration of cationic lipid:pDNA complexes. Hum Gene Ther 10:223-234

22. Moll R, Franke WW, Schiller DL, Geiger B, Krepler R 1982 The catalog of human cytokeratins: patterns of expression in normal epithelia, tumors, and cultured cells. Cell 31:11-24

23. Pankov R, Umezawa A, Maki R, Der CJ, Hauser CA, Oshima RG 1994 Oncogene activation of human keratin 18 transcription via the Ras signal transduction pathway. Proc Natl Acad Sci USA 91:873-877

24. Oshima RG, Abrams L, Kulesh D 1990 Activation of an intron enhancer within the keratin 18 gene by expression of c-fos and c-jun in undifferentiated F9 embryonal carcinoma cells. Genes Dev 4:835-848

25. Neznanov N, Umezawa A, Oshima RG 1997 A regulatory element within a coding exon modulates keratin 18 gene expression in transgenic mice. J Biol Chem 272:27549-27557

26. Chow YH, O'Brodovich H, Plumb J, Wen Y, Sohn KJ, Lu Z, Zhang F, Lukacs GL, Tanswell AK, Hui CC, Buchwald M, Hu J 1997 Development of an epitheliumspecific expression cassette with human DNA regulatory elements for transgene expression in lung airways. Proc Natl Acad Sci USA 94:14695-14700

27. Philip R, Clary B, Brunette E, Kilinski L, Murugesh D, Sorich M, Yau J, Lebkowski J, Lyerly HK, Philip M 1996 Gene modification of primary tumor cells for active immunotherapy of human breast and ovarian cancer. Clin Cancer Res 2:59-68

28. Jobling SA, Gehrke L 1987 Enhanced translation of chimaeric messenger RNAs containing a plant viral untranslated leader sequence. Nature 325:622-625

29. O'Brodovich H, Rafii B, Post M 1990 Bioelectric properties of fetal alveolar epithelial monolayers. Am J Physiol 258:L201-L206

30. Batengurg JJ, Otto-Verburne CJM, Ten Hav-Opbroek AAW, Klazinga K 1988 Isolation of alveolar type II cells from fetal rat lung by differential adherence in monolayer culture. Biochim Biophys Acta 960:441-453

31. Zhang F, Kartner N, Lukacs GL 1998 Limited proteolysis as a probe for arrested conformational maturation of delta F508 CFTR. Nat Struct Biol 5:180-183

32. Kartner N, Augustinas O, Jensen TJ, Naismith AL, Riordan JR 1992 Mislocalization of delta F508 CFTR in cystic fibrosis sweat gland. Nat Genet 1:321-327

33. Carswell S, Alwine JC 1989 Efficiency of utilization of the simian virus 40 late polyadenylation site: effects of upstream sequences. Mol Cell Biol 9:4248-4258

34. Evans MJ, Scarpulla RC 1989 Introns in the $3^{\prime}$-untranslated region can inhibit chimeric CAT and beta-galactosidase gene expression. Gene 84:135-142

35. Fortunati E, Bout A, Zanta MA, Valerio D, Scarpa M 1996 In vitro and in vivo gene transfer to pulmonary cells mediated by cationic liposomes. Biochim Biophys Acta 1306:55-62

36. Low MJ, Goodman RH, Ebert KM 1989 Cryptic human growth hormone gene sequences direct gonadotroph-specific expression in transgenic mice. Mol Endocrinol 3:2028-2033

37. Jacobson A, Peltz SW 1996 Interrelationships of the pathways of mRNA decay and translation in eukaryotic cells. Annu Rev Biochem 65:693-739 